\title{
The technique of robotic lobectomy I: right-sided lobes
}

\author{
Farid Gharagozloo, Mark Meyer \\ Center for Advanced Thoracic Surgery, Global Robotics Institute, Advent Health Celebration, University of Central Florida, \\ Celebration, FL 34786, USA.
}

Correspondence to: Dr. Farid Gharagozloo, Center for Advanced Thoracic Surgery, Global Robotics Institute, Advent Health Celebration University of Central Florida, 400 Celebration Place, Celebration, FL 34786, USA.

E-mail: farid.gharagozloo.md@adventhealth.com

How to cite this article: Gharagozloo F, Meyer M. The technique of robotic lobectomy I: right-sided lobes. Mini-invasive Surg 2020;4:55. http://dx.doi.org/10.20517/2574-1225.2020.42

\author{
Received: 28 Apr 2020 First Decision: 25 May 2020 Revised: 25 May 2020 Accepted: 19 Jun 2020 Published: 1 Sep 2020 \\ Academic Editor: Giulio Belli Copy Editor: Cai-Hong Wang Production Editor: Jing Yu
}

\begin{abstract}
Robotic Lobectomy has been evolving over the past decade and is an oncologically efficacious procedure. Although robotic lobectomy is performed more frequently around the world, it accounts for a small percentage of all lobectomies. The major determinants for the lower level of adoption of the robotic lobectomy procedure are 1. The lack of concise step by step procedure outlines for the surgeons who are transitioning from either open or video-assisted thoracic surgical procedures to robotics, or 2. A strategy for control of catastrophic bleeding during the robotic lobectomy procedure. The Technique of Robotic Lobectomy Part I outlines a stepwise approach to robotic lobectomy for the right upper, middle, and lower lobes. Part II outlines a stepwise approach to robotic lobectomy for left upper, and lower lobes. Part III outlines a methodical technical approach for the control of catastrophic bleeding complications.
\end{abstract}

Keywords: Robotic, lobectomy, bleeding, upper lobectomy, middle lobectomy, lower lobectomy, lung cancer

\section{INTRODUCTION}

The most common indication for lung resection is lung cancer. Approximately 228,150 (116,440 in men and 111,710 in women) new cases of lung cancer were diagnosed in the United States in 2018. During the same period, 142,670 patients died from lung cancer (76,650 in men and 66,020 in women $)^{[1]}$ Lung cancer is by far the leading cause of cancer death among both men and women. Each year, more people die of

cc) (7) (C) The Author(s) 2020. Open Access This article is licensed under a Creative Commons Attribution 4.0 International License (https://creativecommons.org/licenses/by/4.0/), which permits unrestricted use, sharing, adaptation, distribution and reproduction in any medium or format, for any purpose, even commercially, as long as you give appropriate credit to the original author(s) and the source, provide a link to the Creative Commons license, and indicate if changes were made.

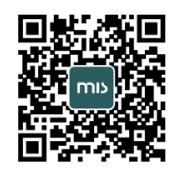


lung cancer than of colon, breast, and prostate cancers combined. The overall five-year survival for lung cancer is approximately $23 \%$. This dismal outlook is due, largely, to the fact that over $50 \%$ of patients are in stage III and IV at the time of diagnosis. On the other hand, the five-year survival for patients with stage I disease is $80 \%-95 \%$. Besides, it is estimated that at any one time approximately 650,000 patients with early lung cancer remain undiagnosed. Lobectomy is indicated as the treatment for early-stage lung cancer.

The first report of the use of the Robotic System for lobectomy in the treatment of primary lung cancer came from Melfi et al. ${ }^{[2]}$ in 2002. Due to safety concerns, the surgery was converted to a thoracotomy in two of five patients. Nevertheless, the report demonstrated the feasibility of the procedure. The first robotic lobectomies were reported in 2003 by Morgan et al. ${ }^{[3]}$ and Ashton et al. ${ }^{[4]}$.

In 2006 Park et al..$^{[5]}$ reported Robot-assisted thoracoscopic Surgery (RATS) technique which represented a hybrid procedure. In 2009, Gharagozloo et al. ${ }^{[6]}$ reported a series of 100 consecutive patients who underwent the RATS procedure which was performed as a hybrid operation using robotic dissection on a video-assisted thoracic surgical (VATS) platform. In the same year, Veronesi et al. ${ }^{[7]}$ described a modified RATS technique with the use of 4 robotic arms. Since that report, the robotic surgical systems and instruments have evolved and the procedures have become more standardized, such that in 2015, over 6000 robotic lobectomies were performed in the United States ${ }^{[8]}$.

Several factors have been responsible for the greater acceptance and use of robotics for lobectomy: (1) introduction of robotic dissecting instruments such as the Endotip Bipolar Dissector (curved tip bipolar), Vessel Sealer, Endowrist Staplers ( curved tip vascular stapler) have facilitated dissection and vascular control; and (2) the newer robotic surgical platform (DaVinci Xi) which has given active control of stapling to the surgeon and relegated the bedside assistant to the more passive role of instrument exchange and specimen retrieval.

More recently, a four-arm completely port-based robotic lobectomy technique (CPRL) was reported by Cerfolio et al. ${ }^{[9]}$. This technique is much simpler and more standardized and can be adapted for all the lobes. It also allows for relatively more efficient use of the assistant port by the bedside surgeon.

CPRL has become the technique of choice for lobectomy as it is completely port-based, uses 4 arms, and $\mathrm{CO}_{2}$ insufflation. Both Si and Xi da Vinci platforms can be used. As described by Cerfolio et al ${ }^{[9]}$, with the da Vinci Si system, the procedure uses three 8- $\mathrm{mm}$ ports (left and right robotic arm ports, fourth robotic arm port), and a 12 -mm port (camera). With the Si system, many surgeons also use a 12 -mm assistant port that can be used for stapling, occasional suction, specimen retrieval, and exchange of items such as rolledup sponges and vessel loops. The assistant port is also important for the management of bleeding in the event of a pulmonary artery or vein injury. With the Xi system, three of the ports are 8-mm ports, and the 4 th is a $12 \mathrm{~mm}$, however, the camera port and the right and left arm ports are $8 \mathrm{~mm}$, and the $12 \mathrm{~mm}$ port is used for the introduction and firing of the robotic Endowrist stapler.

Gharagozloo et al.$^{[10]}$ reported their experience with 638 consecutive robotic lobectomies for early-stage lung cancer. Median operative time was $176 \mathrm{~min}$ (range 160-456), Median Chest tube time was 3 days (2-8 days), Median air leak time was 0 (0-3 days), Median length of stay was 3 days (1-26 days). Minor complications were observed in 133 patients $(21 \%)$. The most common complication was atrial fibrillation which was seen in $13 \%$ of patients. Thirteen $(2.1 \%)$ patients had major complications, including bronchopleural fistula (3), pulmonary embolism (5), acute renal insufficiency (3), hemorrhage (2). Conversion to a thoracotomy occurred in $11(1.7 \%)$ patients. 6/11 conversions were for bleeding. The other conversions (5/11) was due to anatomic and oncologic reasons. There were 3 deaths (0.5\%). All 3 deaths occurred in the first 20 patients and during the learning curve of the procedure. This was attributed to 


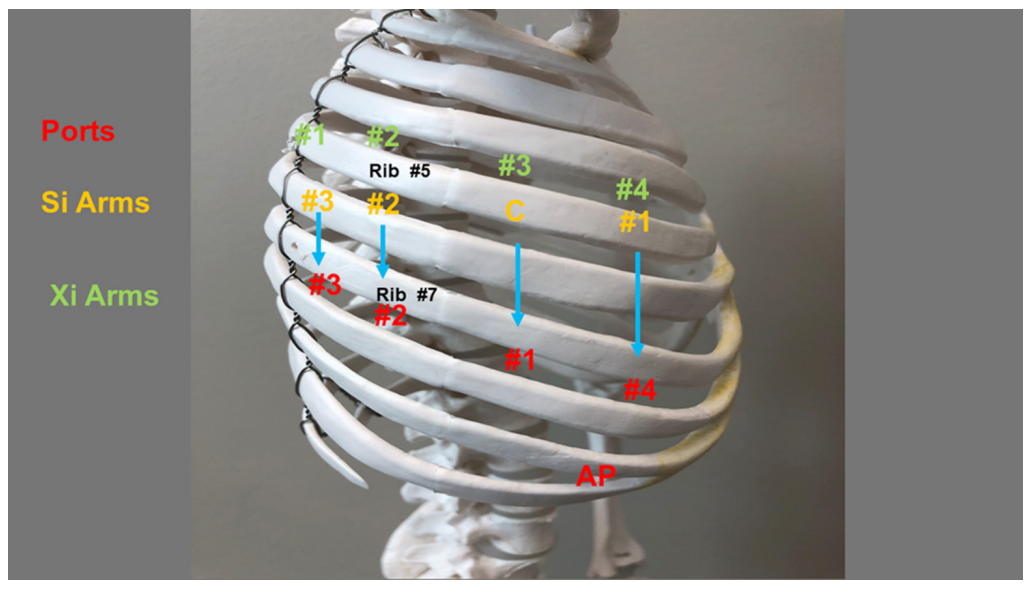

Figure 1. Port placement for robotic lobectomy in the right chest. AP: assistant port

poor patient selection, lack of surgeon and team experience, and rudimentary robotic instruments for the lobectomy procedure. There were no deaths in the last 618 robotic lobectomy procedures. Meyer et al. ${ }^{[11]}$ have reported that based on an assessment which included operative times, surgeon comfort, and mortality, the learning curve of robotic lobectomy was $18 \pm 3$ cases.

The technique of robotic lobectomy is presented in three consecutive papers: Part I outlines a stepwise approach to robotic lobectomy for the right upper, middle and lower lobes. Part II outlines a stepwise approach to robotic lobectomy for left upper, and lower lobes. Part III outlines a methodical technical approach for the control of catastrophic bleeding complications.

\section{RIGHT-SIDED LOBECTOMY}

\section{Port placement}

The operating room table is reversed such that the pedestal does not interfere with the docking of the robot over the head of the patient.

A Double Lumen tube is placed, and the patient is positioned in a full lateral decubitus position. The right arm is placed over pillows and positioned high enough such that access to the 4th intercostal space in the anterior axillary line is readily attained. The table is flexed to move the hip down and to open the intercostal spaces. The lung is deflated and placed on suction. The position of the double-lumen tube is rechecked after the patient is prepped and draped.

Figure 1 shows the right chest port placement. A line is drawn from the tip of the scapula to the costal arch. This delineates the highest point in the chest and the mid scapular line (posterior axillary line). Pleural entry is with a Hassan Needle. Saline is infused and care is taken to look for easy egress of the saline from the needle. If there is any question of pleural adhesions, we use a Visiport Instrument (Medtronic Inc. Norwalk, Conn) for entry into the pleural space under direct vision. If the Visiport is used, a purse-string is placed in the muscle layer and tied around the robot camera port to prevent $\mathrm{CO}_{2}$ leakage.

Port \#1 is the camera port. Warm, humidified $\mathrm{CO}_{2}$ is insufflated through this port at a flow of $6 \mathrm{~L} / \mathrm{min}$ to a pressure of 6-8 $\mathrm{mmHg}$ to push the lung and diaphragm away. The other ports are placed under direct vision. Port \#2 $(8 \mathrm{~mm})$ is placed in the 7 th intercostal space in the poster scapular line. This Port is $9 \mathrm{~cm}$ posterior to Port \#1 and accommodates da Vinci arm \#2. Before the placement of Port \#3, a 21-gauge needle is inserted into the 7th intercostal space at the costovertebral junction from the patient's back and 
injects a $10 \mathrm{~mL}$ subpleural bubble of $0.25 \%$ bupivacaine with epinephrine near the intercostal nerve. Next, Port \#3 is placed $10 \mathrm{~cm}$ posterior to Port \#2 in the 7th intercostal space just medial to the spine. This port accommodates da Vinci arm \#3. Port \#4 is placed $9 \mathrm{~cm}$ anterior to Port \#1 in the 7 th intercostal space at the anterior scapular line. This port accommodates da Vinci arm \#1. The Assistant Port \#5 uses a 10-12 Versiport (Medtronic Inc., Norwalk, Conn, USA) trocar and is placed in the 9th intercostal space and is triangulated between Port \#1 and \#4. It should be two or three ribs lower than and as distant to the da Vinci ports as possible to maximize the assistant workspace. Keeping this port off the trajectory lines for the other ports will facilitate the patient-side assistant's access for the retraction and other maneuvers. In all, including the vitally important Assistant Port, lobectomy is performed with five ports. The use of additional ports should be tailored to the specific situation and the experience of the surgeon. Surgeons are encouraged to use as many ports as are necessary to perform a safe and oncologically efficacious lobectomy.

Port placement and intercostal sites are the same for every lobe. All efforts should be made to keep the distance between the ports as close to what has been described above. In smaller patients, care must be taken to keep the trocar sites as far as possible and within the parameters that have been outlined. This strategy prevents interference in arm function with the present robotic platforms. Port placement may be modified in the future with the development of new platforms and robot arms which may have a smaller "footprint" on the chest.

Port Placement with Si Robot: Robotic arm \#3 is located two cm lateral from the spinous process of the vertebral body, robotic arm $\# 2$ is $10 \mathrm{~cm}$ medial to robotic arm \#3, the camera port (we prefer the $12 \mathrm{~mm}$ camera) is $9 \mathrm{~cm}$ medial to robotic arm 2, and robotic arm \#1 is placed right above the diaphragm anteriorly.

Port Placement with Xi Robot: For the Xi system, the ports are placed in slightly different locations. They are also numbered differently due to the system. Robotic arm \#1 is placed $4 \mathrm{~cm}$ away from the spinous process. Robotic arm \#2 is placed $8 \mathrm{~cm}$ from arm \#1 and robotic arm \#3 is placed $8 \mathrm{~cm}$ from arm \#2. Robotic arm \#4 is placed right above the diaphragm anteriorly. The assistant port is triangulated behind the camera arm and robotic arm \#4 in a similar fashion. The camera is carried by arm \#3. Arms \#1 through \#4 are all placed in the 7 th intercostal space.

Instruments: $0^{\circ}$ and $/$ or $30^{\circ}$ down viewing endoscope, $5 \mathrm{~mm}$ Thoracic Grasper (left (3), Cadiere Forceps (left (2) ) and Curved Bipolar Dissector (right (1) ).

Begin by dividing the inferior pulmonary ligament and remove station \#9, and \#8 nodes [Figures 2 and 3]. Next, the most posterior arm is used to retract the lower lobe medially and anteriorly to remove lymph nodes from station \#7. Next, open the pleura anterior to the vagus nerve and divide the anterior branch of the nerve which traverses the subcarinal space. At the beginning of the case, a nasogastric tube should be inserted to decompress the stomach. After decompression of the stomach, some surgeons may prefer to remove the nasogastric tube to aid in the retraction of the esophagus during the subcarinal dissection. This opens the subcarinal space and allows for better access to the Station \#7 nodes. Identify the right mainstem bronchus and stay posterior to the edge of the cartilage. Remove the station \#7 nodes and control the subcarinal artery at the carina. At the end of the dissection, the right and left mainstem bronchi should be visible, and the posterior aspect of the pericardium should be cleaned and visible [Figure 4]. Next, the most posterior arm is used to retract the upper lobe inferiorly during dissection of stations $2 \mathrm{R}$ and $4 \mathrm{R}$, clearing the space between the superior vena cava (SVC) anteriorly, the trachea posteriorly, and the azygos vein inferiorly [Figure 5].

Completion of the lymph node dissection opens the mediastinal space and facilitates the dissection of the artery and the bronchus. The key to the safe dissection of the posterior aspect of the artery, vein, and 


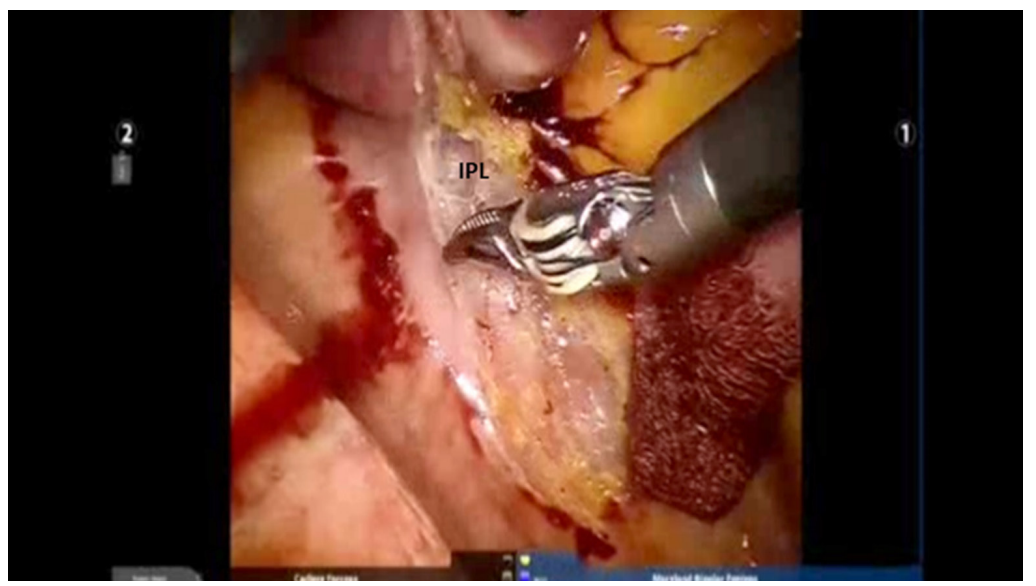

Figure 2. Begin by dividing the IPL. IPL: inferior pulmonary ligament

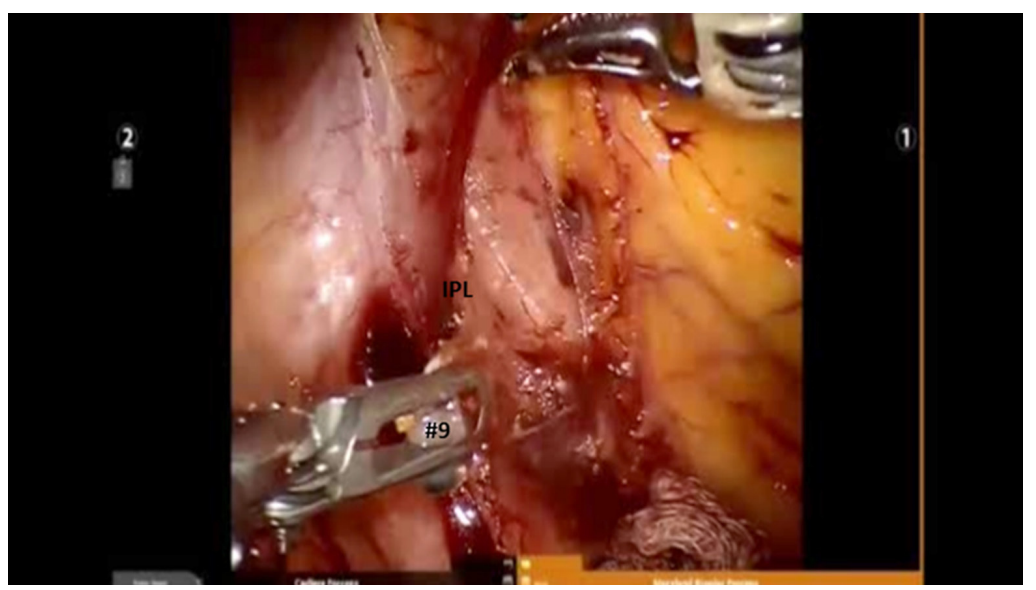

Figure 3. Remove station \# 9, and \#8 nodes. IPL: inferior pulmonary ligament

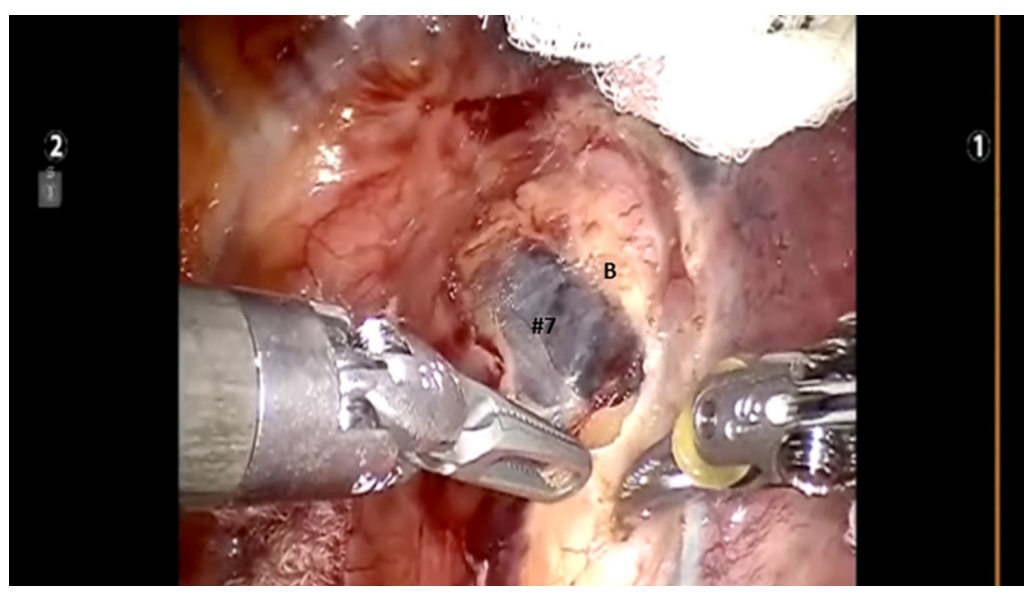

Figure 4. Identify the right mainstem bronchus and stay posterior to the edge of the cartilage. Station \#7 nodal bundle is removed

bronchus is a wide dissection of the mediastinal nodal tissue. The artery, vein, and the bronchus must be encircled under direct vision and after complete skeletonization. All attempts at blunt or blind dissection are strongly discouraged. Not only will blunt dissection without full mobilization of the structure and 


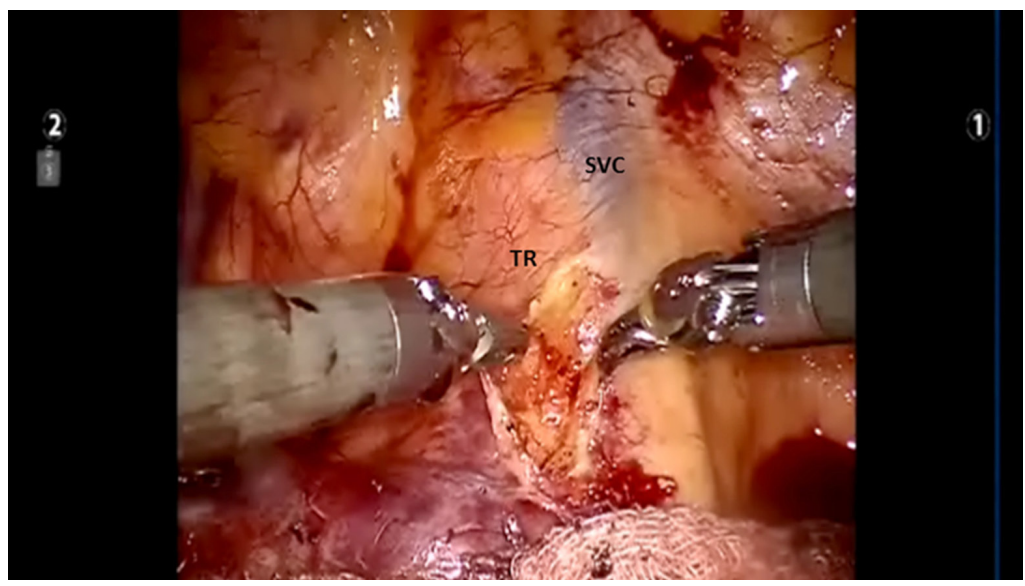

Figure 5. The most posterior arm is used to retract the upper lobe inferiorly during dissection of stations $2 R$ and $4 R$, clearing the space between the SVC anteriorly, the TR posteriorly, and the azygos vein inferiorly. SVC: superior vena cava; TR: trachea

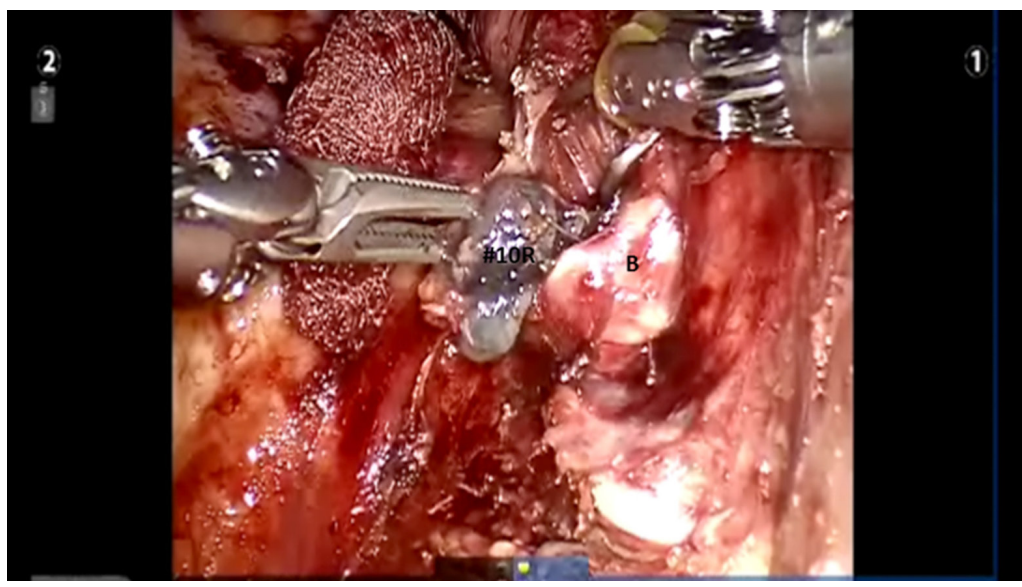

Figure 6. Further dissection of Level \#10 node off the upper lobe bronchus (B)

dissection of the surrounding nodal structures result in injury to these vital structures, but also it will repair the injury extremely difficult. After identifying the right mainstem bronchus, it is followed up to the level of station \#10R lymph node. This node sits between the truncus branch and the superior pulmonary vein. It should be dissected and swept towards the lung, thereby exposing the truncus branch.

Dissection is continued and the crotch between the right upper lobe (RUL) bronchus and bronchus intermedius is defined. All level \#7 nodes and the sump node are removed. This maneuver facilitates later dissection of the pulmonary artery as well as eventual stapling of the RUL bronchus [Figure 6].

Next, the lung is retracted posteriorly to expose the anterior hilum. The dissection is carried down between the hilar structures and the phrenic nerve. The phrenic nerve is swept down to remove the \#10R lymph node. The bifurcation between the middle and upper lobe veins is dissected. It is best to encircle the entire upper lobe vein off the underlying pulmonary artery using the Cadiere Forceps in the left arm and pass a red rubber vessel loop to elevate the vein. This makes the dissection of the middle lobe vein easier. Following the dissection of the middle lobe vein, the Cadiere Forceps is passed under the elevated upper lobe vein, the vessel loop is released and re-grasped thereby isolating the upper lobe vein. Dissection is continued and the proximal main pulmonary artery is exposed as it emerges from the pericardium. The upper lobe vein is divided using a vascular stapler either using the robot arm or passed through the 


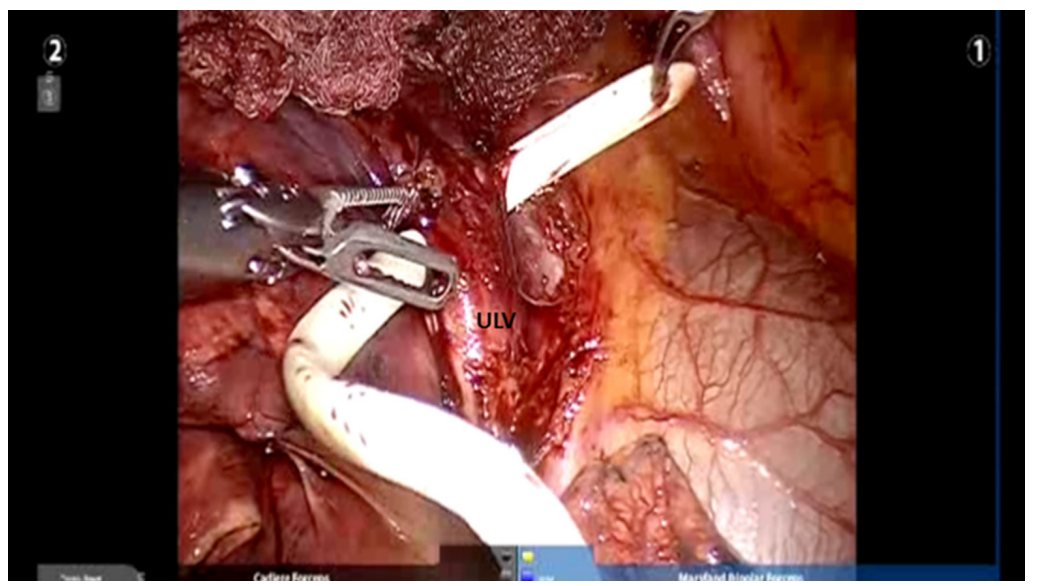

Figure 7. The ULV is elevated, the leader catheter portion of a vascular stapler is passed underneath. ULV: upper lobe vein

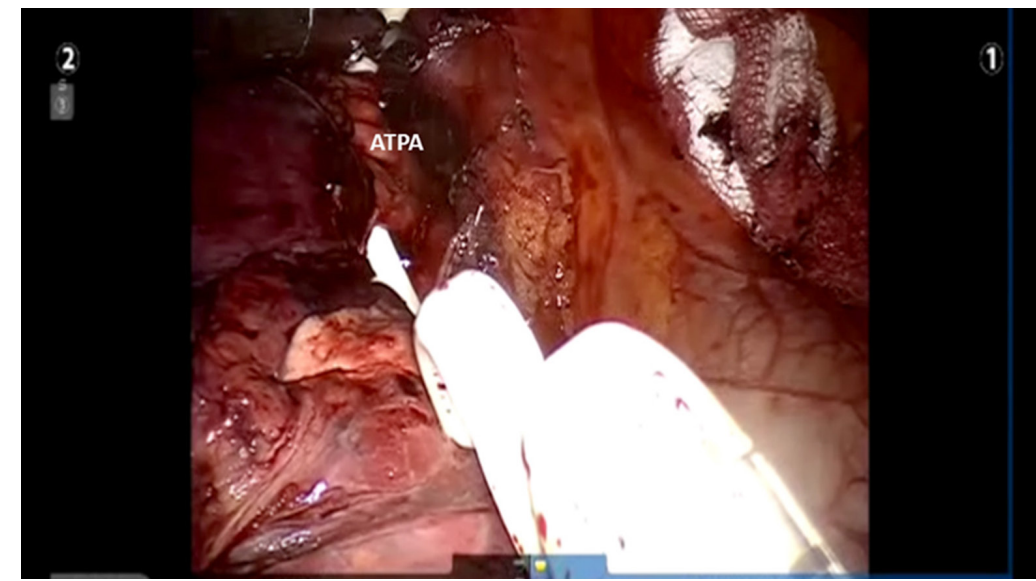

Figure 8. The anterior ATPA branch is elevated, encircled, and the leader catheter portion of a vascular stapler is used to position the stapler. ATPA: apical trunk pulmonary artery

accessory port by the assistant (Covidien Signia gold tip with leader catheter with white 45 cartridges) [Figure 7].

Next, the anterior apical trunk pulmonary artery branch is encircled with a vessel loop and transected with a linear stapler in the same fashion as the vein [Figure 8].

The Lung is once again reflected anteriorly, thereby exposing the posterior aspect of the hilum. The right upper lobe bronchus is identified from a posterior approach and it is separated from the main pulmonary artery which lies underneath. The RUL bronchus is encircled with a vessel loop and transected using a stapler with a green cartridge. Before firing the stapler, the anesthesiologist must make certain that the suction catheter in the endotracheal tube is removed [Figure 9].

After this maneuver, the posterior segmental pulmonary artery is exposed and divided. This vessel is usually smaller than $6 \mathrm{~mm}$ and therefore can be divided with a vessel-sealing device and the proximal stump is further reinforced with a small Titanium clip applied with the robotic Endowrist small clip applier.

Next, the fissure between the right upper lobe and the right middle lobe is divided with gold or purple stapler from an anterior to posterior direction [Figure 10]. Finally, the lung is retracted superiorly, the 


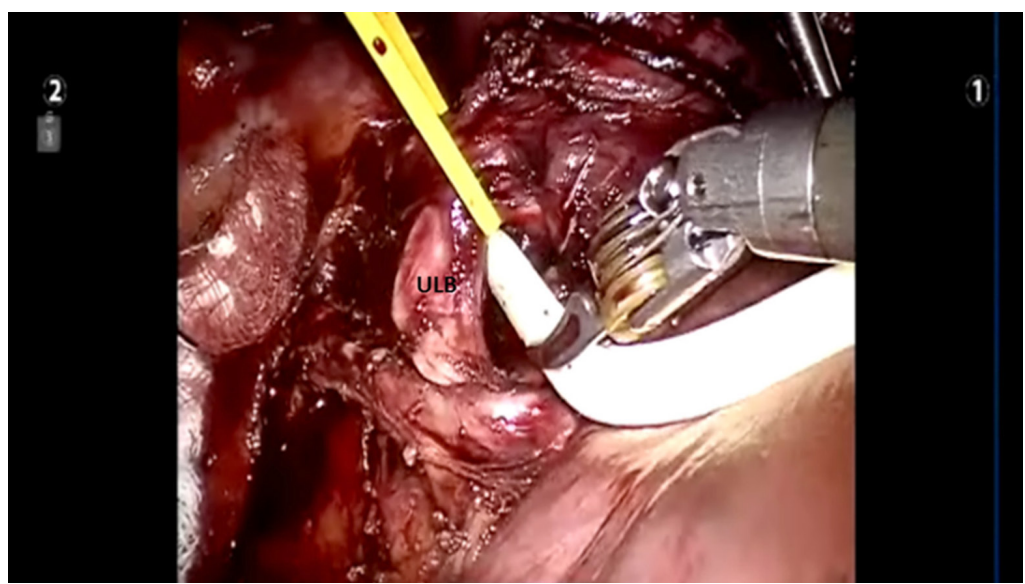

Figure 9. The right upper lobe bronchus is encircled with a vessel loop before passage of the stapler. ULB: upper lobe bronchus

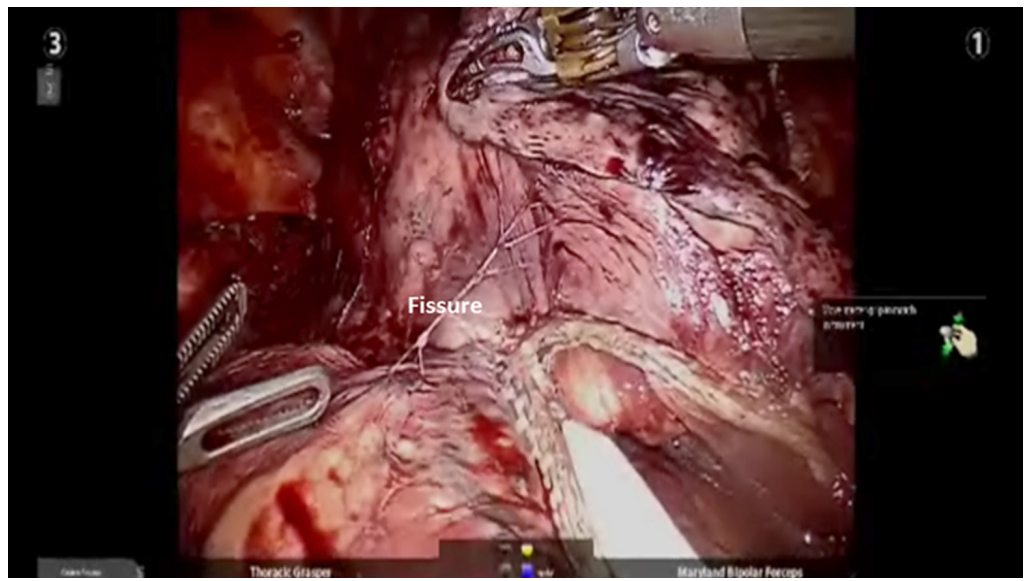

Figure 10. Divided transverse fissure

pulmonary artery is visualized, and the posterior aspect of the fissure (minor fissure) is divided in a similar manner.

The specimen retrieval bag (Anchor Tissue Retrieval System, ConMed Inc. Utica, NY, USA) is introduced through the Accessory port, the specimen is placed into the bag, and the bag is removed.

The dissection begins like that of upper lobectomy by dividing the inferior pulmonary vein and removing station \#9, \#8, and \#7 nodes. Next, the most posterior arm is used to retract the upper lobe inferiorly during dissection of stations $2 \mathrm{R}$ and $4 \mathrm{R}$, clearing the space between the SVC anteriorly, the trachea posteriorly, and the azygos vein inferiorly. Completion of the lymph node dissection opens the mediastinal space and facilitates the dissection of the artery and the bronchus.

The pleura posterior to the phrenic nerve is incised. The superior pulmonary vein is dissected in the same manner as with right upper Lobectomy. The bifurcation between the right upper and middle lobar veins is developed by dissecting it off the underlying pulmonary artery. The right middle lobe vein is encircled and divided [Figure 11].

In our experience, the best way to enter the appropriate plane over the pulmonary artery is to follow the anterior segmental branch to the lower lobe. This branch is usually very superficial and is not covered with 


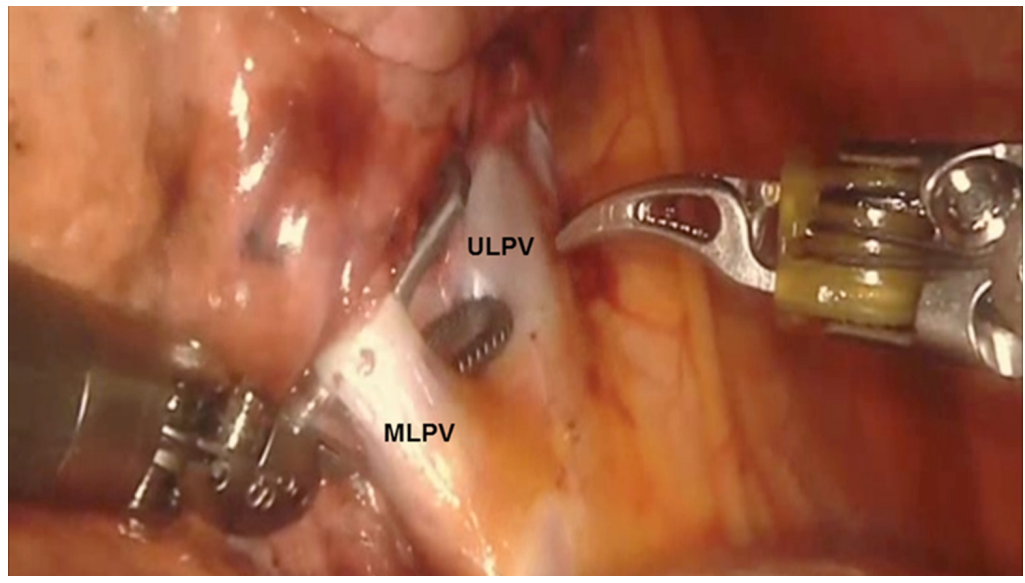

Figure 11. The right MLPV is encircled. ULPV is seen. MLPV: middle lobe pulmonary vein; ULPV: upper lobe pulmonary vein

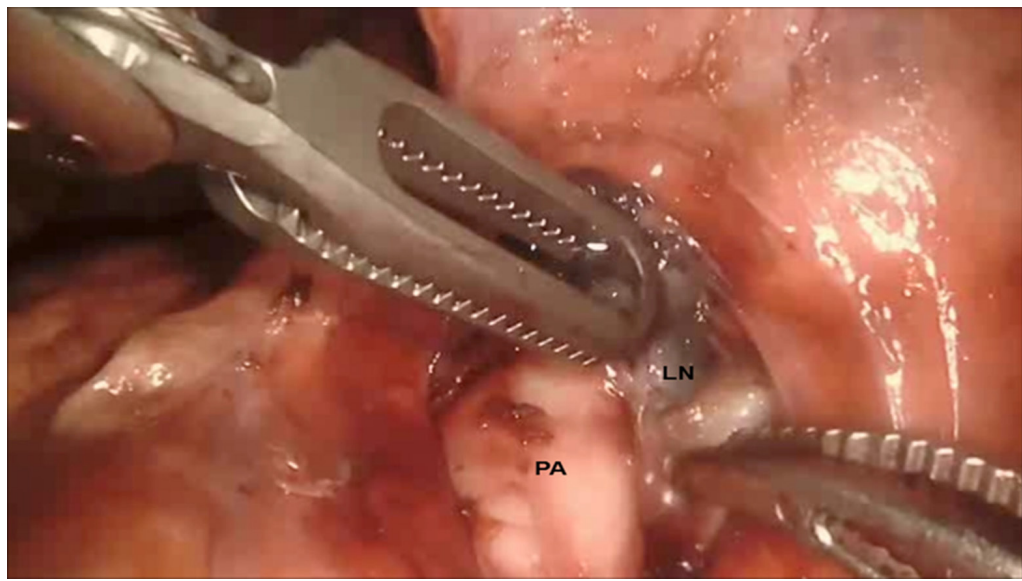

Figure 12. Removal of station \#11 nodes off of the pulmonary artery in the fissure. PA: pulmonary artery; LN: lymph node station \#11

nodal or parenchymal tissue. This branch can be followed superiorly to the main pulmonary artery. This maneuver helps to elevate station \#11 nodes off the pulmonary artery and to identify the artery branch to the middle lobe. Next, the remainder of the fissure between the right middle lobe and right lower lobe is divided in an anterior to posterior direction [Figure 12]. At times there is a vein branch to the middle lobe which drains into the inferior pulmonary vein. This is divided into the remainder of the anterior fissure.

Next, the middle lobe bronchus is identified. It will be running from left to right in the fissure. It is encircled and divided, taking care to avoid injuring the pulmonary artery branches that are located directly behind it [Figure 13].

The middle lobe artery is encircled and divided with a vascular load. At times right middle lobe artery branches come off directly from the main pulmonary artery instead of bifurcating from the common trunk of a single middle lobe artery. These are encircled and divided in the same fashion [Figure 14].

Dissection of the fissure is then continued posteriorly until the main artery trunk and the superior segmental artery branch are identified. After identifying the main artery, the Cadiere forceps in the left hand is used to go under the transverse fissure in a posterior to anterior direction heading for the divided superior pulmonary vein. A vessel loop is passed, and the fissure between the upper and middle lobes is 


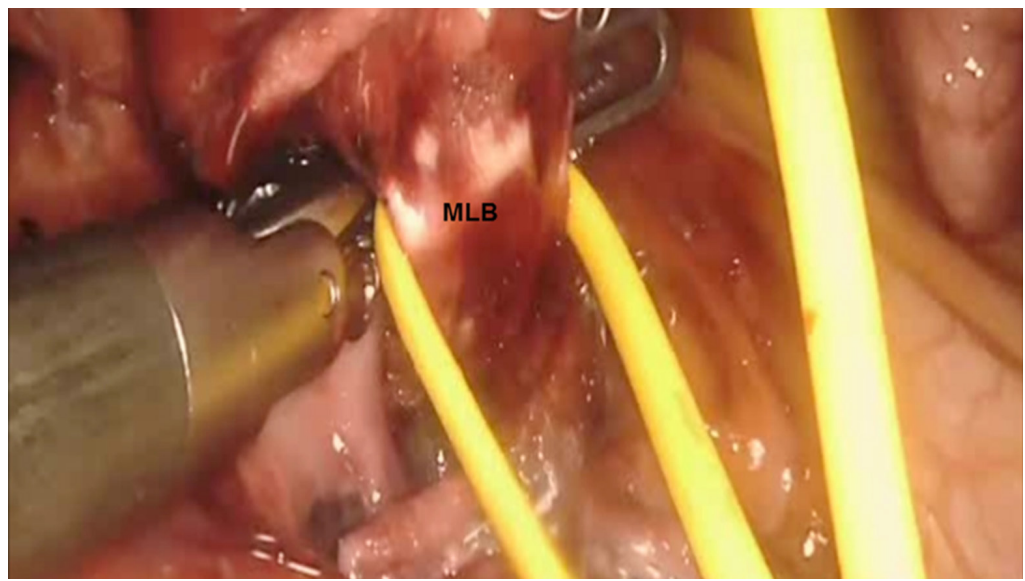

Figure 13. A vessel loop is passed around the MLB and used to elevate it off the pulmonary artery. MLB: middle lobe bronchus

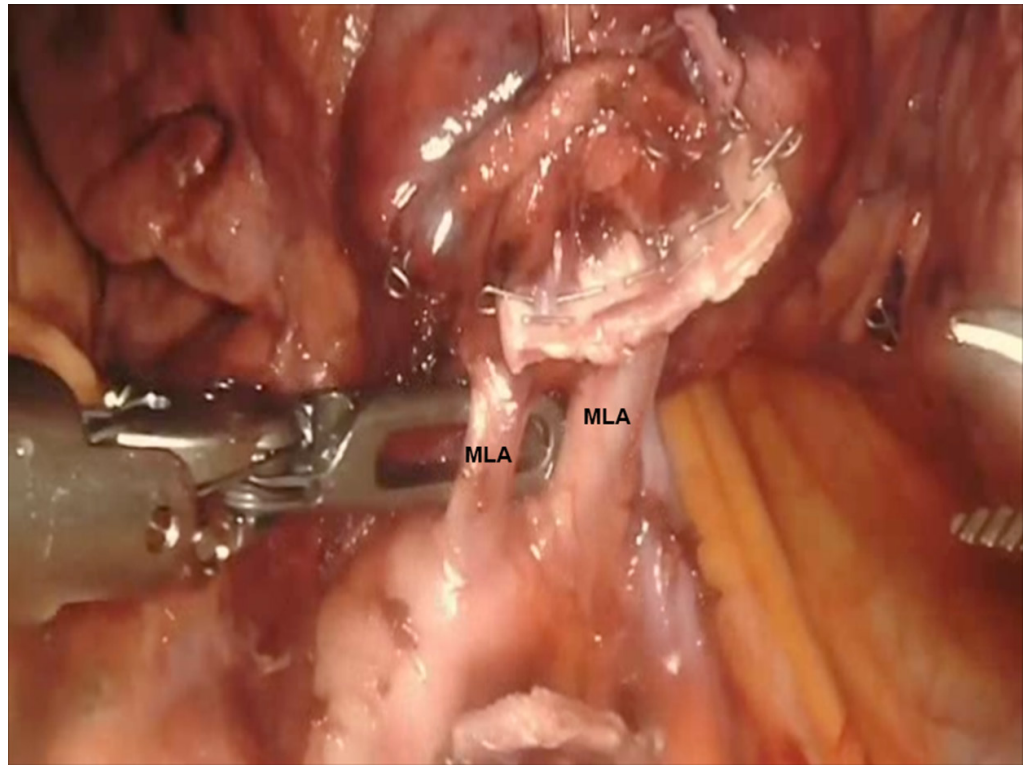

Figure 14. Right middle lobe artery branches (RML) are encircled. MLA: right middle lobe artery branch.

divided using a stapler [Figure 15].

The docking, setup and mediastinal nodal dissection are similar to Right Upper Lobectomy.

Following the mediastinal nodal dissection, the lung is retracted posteriorly and held in place with the robot arm. The bifurcation of the right superior and inferior pulmonary veins is dissected and delineated. The location of the right middle lobar vein should be positively identified to avoid inadvertent transection. The inferior pulmonary vein is encircled using the Cadiere Forceps and divided using a white vascular cartridge.

The anterior branch of the lower lobe pulmonary artery is most superficial and usually does not have overlying nodal tissue. This branch is identified and traced back to the main trunk of the pulmonary artery. Next, the sub adventitial plane overlying the pulmonary artery is developed and nodal tissue (Station \#11) is removed. Retraction is released and the lung is allowed to remain in its normal position, thereby facilitating visualization of the oblique fissure. The dissection is carried posteriorly in the sub adventitial 


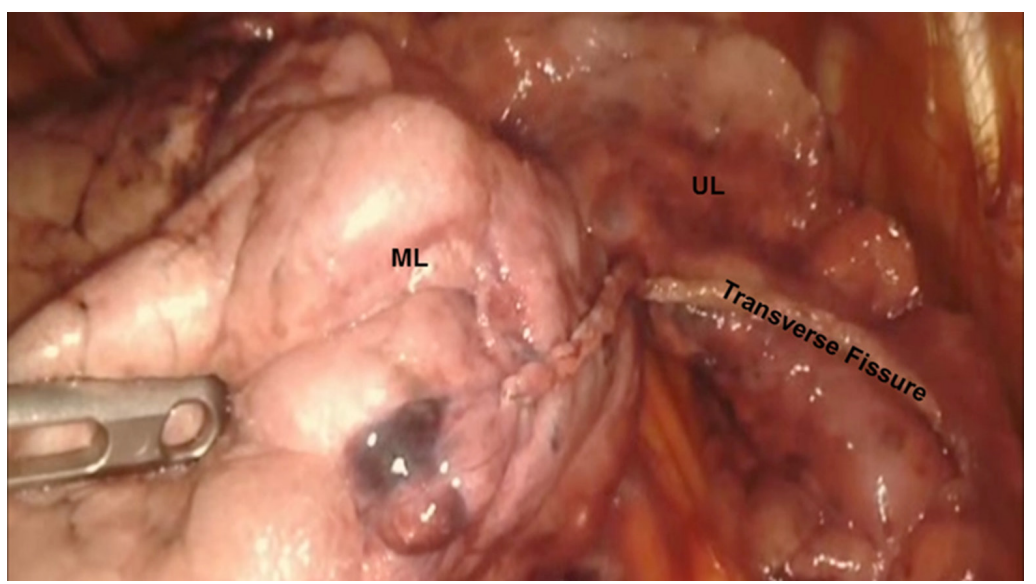

Figure 15. View of the divided transverse fissure. UL: upper lobe; ML: middle lobe

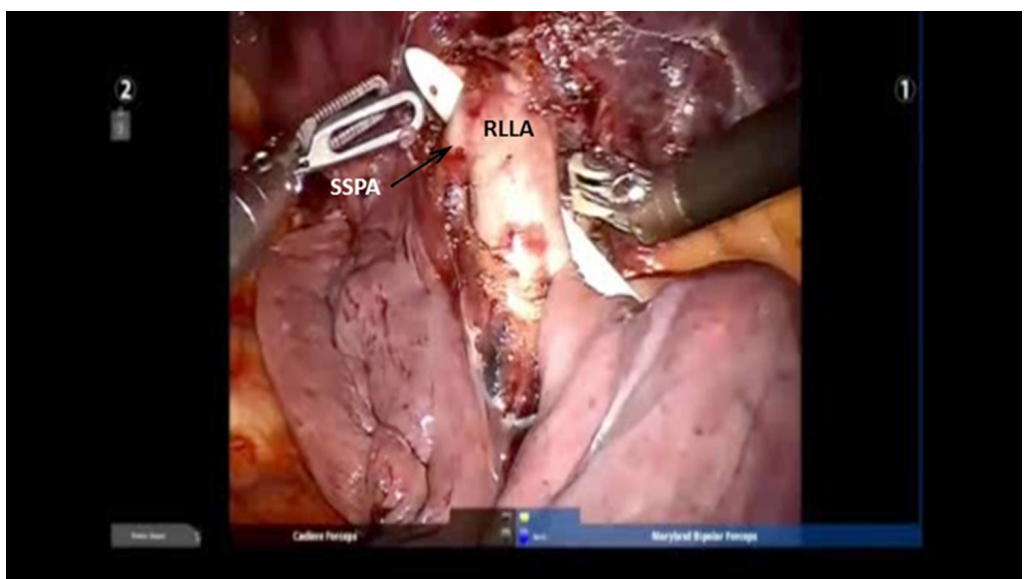

Figure 16. The pulmonary artery branch to the lower lobe (RLLA) is identified. The SSPA is identified. RLLA: right lower lobe pulmonary artery; SSPA: superior segmental artery

layer and the superior segmental branch of the lower lobe pulmonary artery is identified. The major fissure is then divided from an anterior to posterior direction using a stapler which is introduced from the anterior port.

The pulmonary artery branch to the lower lobe is identified. At times the superior segmental pulmonary artery and the inferior lower lobe segmental artery can be taken by encircling the pulmonary artery proximal to the takeoff of the superior segmental artery. Other times these branches need to be taken separately. We prefer to take the inferior segmental artery first, thereby making the encirclement of the superior segmental artery easier [Figure 16].

Next, the posterior aspect of the oblique fissure is divided using a stapler with a purple cartridge. Finally, the bronchus is encircled and divided using a purple cartridge [Figure 17]. The lower lobe is removed as described earlier.

\section{CONCLUSION}

Robotic Lobectomy has been evolving over the past decade and is an oncologically acceptable procedure. A methodical approach to the conduct of the lobectomies and a proven strategy for the control of major vascular injury will increase adoption. The technique of Robotic Lobectomy Part II outlines the technique 


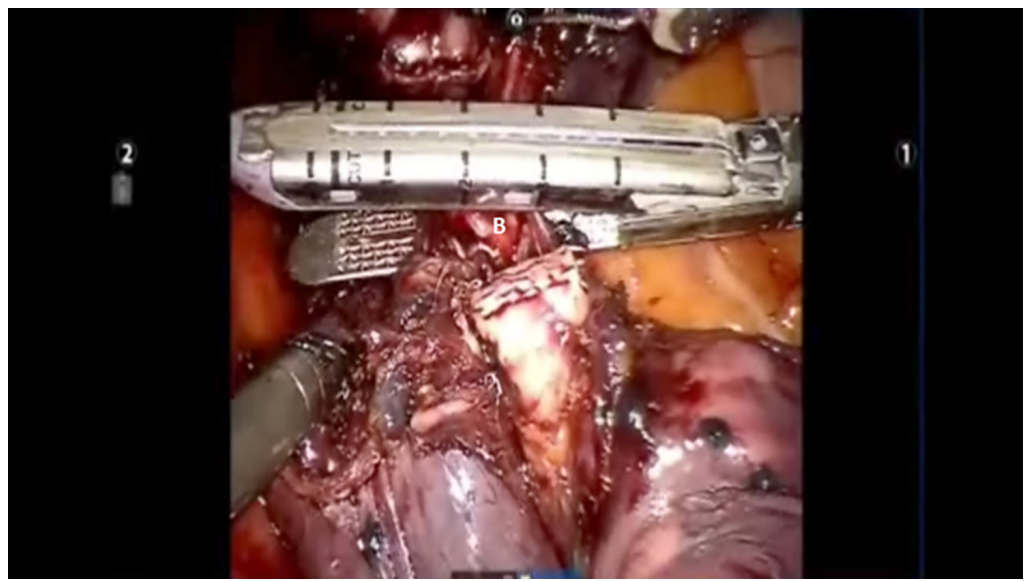

Figure 17. The lower lobe bronchus (B) is divided using with a stapler holding a purple cartridge

of lobectomy for the left-sided lobes. The technique of Robotic Lobectomy Part III outlines the methodical approach for the control of catastrophic bleeding complications.

\section{DECLARATIONS}

\section{Authors' contributions}

Participated in the research, performed the procedures and wrote the manuscript: Gharagozloo F, Meyer M

\section{Availability of data and materials}

Not applicable.

\section{Financial support and sponsorship}

None.

\section{Conflicts of interest}

All authors declared that there are no conflicts of interest.

\section{Ethical approval and consent to participate}

Not applicable.

\section{Consent for publication}

Not applicable.

\section{Copyright}

(c) The Author(s) 2020.

\section{REFERENCES}

1. American Cancer Society: Cancer Facts and Figures-2018. Atlanta, American Cancer Society. Available from: https://www.cancer.org/ research/cancer-facts-statistics/all-cancer-facts-figures/cancer-facts-figures-2018.html. [Last accessed on 3 Jul 2020]

2. Melfi FM, Menconi GF, Mariani AM, Angeletti CA. Early experience with robotic technology for thoracoscopic surgery. Eur J Cardiothoracic Surg 2002;21:864-8.

3. Morgan JA, Ginsburg ME, Sonett JR, Morales DL, Kohmoto T, et al. Advanced thoracoscopic procedures are facilitated by computeraided robotic technology. Eur J Cardiothorac Surg 2003;23:883-7.

4. Ashton RC Jr, Connery CP, Swistel DG, DeRose JJ Jr. Robot-assisted lobectomy. J Thorac Cardiovasc Surg 2003;126:292-3.

5. Park BJ, Flores RM, Rusch VW. Robotic assistance for video-assisted thoracic surgical lobectomy: technique and initial results. J Thorac Cardiovasc Surg 2006;131:54-9. 
6. Gharagozloo F, Margolis M, Tempesta B, Strother E, Najam F. Robot-assisted lobectomy for early-stage lung cancer: report of 100 cases. Ann Thorac Surg 2009;88:380-4.

7. Veronesi G, Agoglia BG, Melfi F, Maisonneuve P, Bertolotti R, et al. Experience with robotic lobectomy for lung cancer. Innovations (Phila) 2011;6:355-60.

8. Cerfolio RJ. Total port approach for robotic lobectomy. Thorac Surg Clin 2014;24:151-6.

9. Cerfolio RJ, Bryant AS, Skylizard L, Minnich DJ. Initial consecutive experience of completely portal robotic pulmonary resection with 4 arms. J Thorac Cardiovasc Surg 2011;142:740-6.

10. Gharagozloo F, Meyer M, Tempesta B. Robotic lobectomy: experience with 638 consecutive cases. Surg Tech Int 2020;36:251-6.

11. Meyer M, Gharagozloo F, Tempesta B, Margolis M, Strother E, et al. The learning curve of robotic lobectomy. Int J Med Robot 2012;8:448-52. 